\title{
An Intramolecular Hetero Diels-Alder (Povarov) Approach to the Synthesis of the Alkaloids Luotonin A and Camptothecin
}

\author{
Heather Twin and Robert A. Batey
}

\section{Experimental Procedure}

\section{General Synthetic Methods}

THF, hexanes, benzene and toluene were distilled from sodium metal/benzophenone ketyl under argon. $\mathrm{CH}_{2} \mathrm{Cl}_{2}$ and $\mathrm{MeCN}$ were distilled from $\mathrm{CaH}_{2}$ under argon. All other solvents were obtained as ACS grade from commercial suppliers and used directly. All reagents, unless otherwise stated, were used as received from commercial suppliers (Aldrich, Fischer, Strem or Lancaster). All glassware, unless otherwise stated, was flame dried under a stream of dry nitrogen. All reactions, unless stated otherwise, were performed under an atmosphere of dry nitrogen. Melting points were obtained on a Fisher-Johns melting point apparatus and are uncorrected. FT-IR spectra were obtained on a Perkin-Elmer Spectrum 1000 spectrometer, with samples loaded as films on $\mathrm{NaCl}$ plates. ${ }^{1} \mathrm{H}$ and ${ }^{13} \mathrm{C}$ NMR spectra were obtained on Varian Mercury 300 or Unity 400 or 500 spectrometers as solutions in deuterated solvents (normally $\mathrm{CDCl}_{3}$ or $\mathrm{CD}_{3} \mathrm{OD}$, obtained from Cambridge Isotope Labs) and referenced to their corresponding solvent peaks (e.g., $\mathrm{CDCl}_{3}, \delta 7.26 \mathrm{ppm} ; \mathrm{CD}_{3} \mathrm{OD}, \delta 3.31 \mathrm{ppm}$ for proton resonances and $\mathrm{CDCl}_{3}, \delta$ $77.23 \mathrm{ppm} ; \mathrm{CD}_{3} \mathrm{OD}, \delta 49.15 \mathrm{ppm}$; for carbon resonances). Low-resolution mass spectra (MS) were recorded on a Bell and Howell 21-490 spectrometer and high-resolution mass spectra (HRMS) were recorded on an AEI MS3074 spectrometer. Flash column chromatography on silica gel (60 A, 230-400 mesh, low acidity, obtained from Silicycle Inc.) was performed with reagent grade solvents. Analytical thin-layer chromatography (TLC) was performed on precoated silica gel plates (Alugram SIL G/UV ${ }_{254}$ purchased from Rose Scientific Ltd or Silicycle Inc.), visualized with a $\mathrm{UV}_{254}$ lamp (Spectroline, Longlife filter) and stained with $20 \%$ ninhydrin or $\mathrm{KMnO}_{4}$. 
6-Dimethoxymethyl-4-methyl-2-oxo-1,2-dihydropyridine-3-carbonitrile 5<smiles>COC(C)C(C)=O</smiles><smiles>COC(C)(C)N(C)C</smiles><smiles>N#CCC(N)=O</smiles><smiles>COC(OC)c1cc(C)c(C#N)c(=O)[nH]1</smiles>

Pyruvic aldehyde dimethylacetal $(3.63 \mathrm{~mL}, 0.03 \mathrm{~mol})$ and dimethylformamide dimethylacetal $(7.31 \mathrm{~mL}, 0.45 \mathrm{~mol})$ in anhydrous DMF $(20 \mathrm{~mL})$ were heated at $80^{\circ} \mathrm{C}$ for 3 hours, after which time, the solvent was removed under reduced pressure. A solution of the crude enamine in DMF $(10 \mathrm{~mL})$ was added dropwise to a stirred solution containing sodium methoxide $(3.56 \mathrm{~g}, 0.07$ mol) and 2-cyanoacetamide $(2.77 \mathrm{~g}, 0.03 \mathrm{~mol})$ in DMF $(40 \mathrm{~mL})$. The resulting mixture was heated at $100{ }^{\circ} \mathrm{C}$ for 3 hours and then concentrated under reduced pressure. $\mathrm{CH}_{2} \mathrm{Cl}_{2}(100 \mathrm{~mL})$ and water $(60 \mathrm{~mL})$ were added to the residue, and the $\mathrm{pH}$ adjusted to 7 by the addition of $\mathrm{HCl}$ (1 $\mathrm{M}$ soln.). The $\mathrm{CH}_{2} \mathrm{Cl}_{2}$ layer was separated and the aqueous phase was extracted with $\mathrm{CH}_{2} \mathrm{Cl}_{2}(4 \mathrm{x}$ $100 \mathrm{~mL})$. The combined organic extracts were washed with brine $(1 \times 50 \mathrm{~mL})$, dried $\left(\mathrm{MgSO}_{4}\right)$ and concentrated under reduced pressure. The crude product was recrystallized (EtOAc/Hexanes) to give 6-dimethoxymethyl-4-methyl-2-oxo-1,2-dihydropyridine-3-carbonitrile $5(1.80 \mathrm{~g}, 29 \%)$ as a pale yellow solid. m.p. $=197-199^{\circ} \mathrm{C} ; \mathrm{R}_{f}=0.03$ (50\% EtOAc:Hexanes); IR (film) $3218,1651,1385,1202,1114,913 \mathrm{~cm}^{-1} ; \delta_{\mathrm{H}}\left(300 \mathrm{MHz}, \mathrm{CDCl}_{3}\right) 6.33(1 \mathrm{H}, \mathrm{s}), 5.30(1 \mathrm{H}, \mathrm{s})$, $3.45(6 \mathrm{H}, \mathrm{s}), 2.48(3 \mathrm{H}, \mathrm{s}) ; \delta_{\mathrm{C}}\left(75 \mathrm{MHz}, \mathrm{CDCl}_{3}\right)$ 161.4, 161.0, 147.6, 114.7, 106.4, 104.4, 98.3, 53.9, 21.5; MS (EI) m/e (rel intensity) 208 (39\%), 177 (100\%), 119 (51\%), 75 (94\%); HRMS (EI) $m / e \mathrm{C}_{10} \mathrm{H}_{12} \mathrm{~N}_{2} \mathrm{O}_{3}$ calcd. 208.0848, found 208.0844.

6-Dimethoxymethyl-4-methyl-2-oxo-1-prop-2-ynyl-1,2-dihydropyridine-3-carbonitrile<smiles>COC(OC)c1cc(C)c(C#N)c(=O)[nH]1</smiles><smiles>C#CCn1c(C(OC)OC)cc(C)c(C#N)c1=O</smiles>

To a suspension of 6-dimethoxymethyl-4-methyl-2-oxo-1,2-dihydropyridine-3-carbonitrile 5 $(400 \mathrm{mg}, 1.92 \mathrm{mmol})$ in a mixture of water $(0.15 \mathrm{~mL})$ and toluene $(15 \mathrm{~mL})$ was added potassium carbonate (531 mg, $3.84 \mathrm{mmol})$, lithium bromide (334 mg, $3.84 \mathrm{mmol})$, TBAB (61 mg, 0.19 
mmol) and propargyl bromide $(321 \mu \mathrm{L}, 2.88 \mathrm{mmol}, 80 \%$ soln. in toluene). The reaction mixture was heated for 1 hour at $100{ }^{\circ} \mathrm{C}$. The inorganic residues were removed by filtration over Celite, washing thoroughly with $\mathrm{CH}_{2} \mathrm{Cl}_{2}$ and the filtrate concentrated under reduced pressure. Flash chromatography (20\% EtOAc:Hexanes) gave 6-dimethoxymethyl-4-methyl-2-oxo-1-prop-2ynyl-1,2-dihydropyridine-3-carbonitrile (404 mg, 86\%) as a pale yellow solid. m.p. $=78-80{ }^{\circ} \mathrm{C}$; $\mathrm{R}_{f}=0.23$ (50\% EtOAc:Hexanes); IR(film) 3306, 2940, 2253, 2228, 1655, 1604, 1552, 1450, 1389, 1353, 1187, 1124, 1063, $912 \mathrm{~cm}^{-1} ; \delta_{\mathrm{H}}\left(500 \mathrm{MHz}, \mathrm{CDCl}_{3}\right) 6.49(1 \mathrm{H}, \mathrm{s}), 5.46(1 \mathrm{H}, \mathrm{s}), 5.00$ $(2 \mathrm{H}, \mathrm{d}, J=2.5 \mathrm{~Hz}), 3.40(6 \mathrm{H}, \mathrm{s}), 2.46(3 \mathrm{H}, \mathrm{s}), 2.29(1 \mathrm{H}, \mathrm{t}, J=2.5 \mathrm{~Hz}) ; \delta_{\mathrm{C}}\left(125 \mathrm{MHz}, \mathrm{CDCl}_{3}\right)$ 159.8, 158.6, 146.6, 114.6, 108.3, 104.8, 98.4, 77.4, 72.5, 53.7, 33.2, 21.3; MS (EI) m/e (rel intensity) 246 (29\%), 231 (100\%), 201 (19\%), 185 (17\%); HRMS (EI) m/e $\mathrm{C}_{13} \mathrm{H}_{14} \mathrm{~N}_{2} \mathrm{O}_{3}$ calcd. 246.1004, found 246.1002 .

\section{$\underline{\text { 6-Formyl-4-methyl-2-oxo-1-prop-2-ynyl-1,2-dihydropyridine-3-carbonitrile } 6}$}
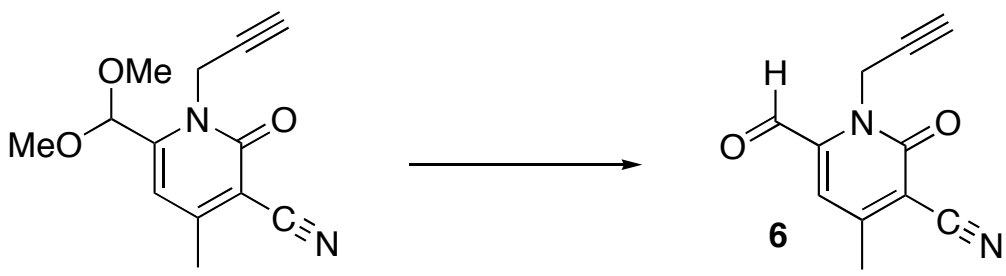

To 6-dimethoxymethyl-4-methyl-2-oxo-1-prop-2-ynyl-1,2-dihydropyridine-3-carbonitrile (74 $\mathrm{mg}, 0.30 \mathrm{mmol})$ was added a mixture of $\mathrm{HCl}(0.5 \mathrm{~mL}$, conc. $)$, glacial acetic acid (1.5 mL) and water $(2.5 \mathrm{~mL})$. The reaction was heated for 1 hour at $60{ }^{\circ} \mathrm{C}$ then allowed to cool to room temperature. After being poured into water $(20 \mathrm{~mL}), \mathrm{NaHCO}_{3}(20 \mathrm{~mL}$ sat. aq. soln. $)$ and ethyl acetate $(4 \times 20 \mathrm{~mL})$ were added. The combined organic extracts were washed with brine $(2 \times 10$ $\mathrm{mL}$, sat. aq. soln.), dried over $\mathrm{MgSO}_{4}$, filtered and the solvent removed in vacuo to give 6formyl-4-methyl-2-oxo-1-prop-2-ynyl-1,2-dihydropyridine-3-carbonitrile 6 (57 mg, 95\%) as a white solid. m.p. $=174-176{ }^{\circ} \mathrm{C}$; IR (film) $3422,1638,1266 \mathrm{~cm}^{-1} ; \delta_{\mathrm{H}}\left(300 \mathrm{MHz}, \mathrm{CDCl}_{3} / \mathrm{CD}_{2} \mathrm{Cl}_{2}\right)$ $9.74(1 \mathrm{H}, \mathrm{s}), 6.79(1 \mathrm{H}, \mathrm{s}), 5.28(2 \mathrm{H}, \mathrm{d}, J=2.0 \mathrm{~Hz}), 2.57(3 \mathrm{H}, \mathrm{s}), 2.29(1 \mathrm{H}, \mathrm{t}, J=2.5 \mathrm{~Hz}) ; \delta_{\mathrm{C}}(75$ $\mathrm{MHz}, \mathrm{CDCl}_{3} / \mathrm{CD}_{2} \mathrm{Cl}_{2}$ ) (1 carbon missing) 184.1, 158.5, 157.9, 141.0, 119.4, 113.7, 77.3, 73.1, 32.8, 21.1; MS (EI) m/e (rel intensity) 200 (61\%), 172 (100\%), 143 (50\%); HRMS (EI) m/e $\mathrm{C}_{11} \mathrm{H}_{8} \mathrm{~N}_{2} \mathrm{O}_{2}$ calcd. 200.0586, found 200.0583. 


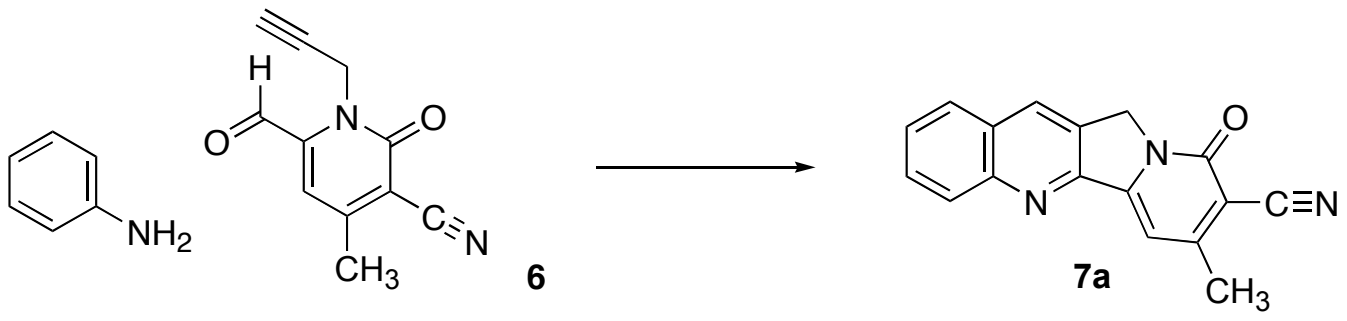

To 6-formyl-4-methyl-2-oxo-1-prop-2-ynyl-1,2-dihydropyridine-3-carbonitrile 6 (40 mg, 0.20 $\mathrm{mmol})$ in acetonitrile $(10 \mathrm{~mL})$ was added aniline $(18 \mu \mathrm{L}, 0.20 \mathrm{mmol})$ followed by Dy $(\mathrm{OTf})_{3}(12$ $\mathrm{mg}, 0.02 \mathrm{mmol}$ ). The reaction mixture was heated at $50{ }^{\circ} \mathrm{C}$ for 16 hours, then allowed to cool. Removal of the solvent followed by recrystallization from hot methanol gave 7-methyl-9-oxo9,11-dihydroindolizino[1,2-b]quinoline-8-carbonitrile 7a (39 $\mathrm{mg}, 71 \%$ ) as a fine yellow powder. m.p. $\geq 300{ }^{\circ} \mathrm{C} ; \delta_{\mathrm{H}}\left(300 \mathrm{MHz}, \mathrm{CD}_{2} \mathrm{Cl}_{2}+\mathrm{CD}_{3} \mathrm{OD}\right) 8.50(1 \mathrm{H}, \mathrm{s}), 8.21(1 \mathrm{H}, \mathrm{d}, J=9.0 \mathrm{~Hz}), 8.00(1 \mathrm{H}$, d, $J=8.5 \mathrm{~Hz}), 7.86(1 \mathrm{H}, \mathrm{ddd}, J=8.5,7.0,1.5 \mathrm{~Hz}), 7.71(1 \mathrm{H}, \mathrm{ddd}, J=8.0,7.0,1.0 \mathrm{~Hz}), 7.32(1 \mathrm{H}$, s), $5.30(2 \mathrm{H}, \mathrm{s}), 2.64(3 \mathrm{H}, \mathrm{s}) ; \delta_{\mathrm{C}}\left(75 \mathrm{MHz}, \mathrm{CDCl}_{3}+\mathrm{CD}_{3} \mathrm{OD}\right)$ 162.5, 150.9, 148.8, 131.7, 131.0, 129.2, 129.0, 128.6, 128.2, 103.2, 77.2, 50.4, 21.3; MS (EI) m/e (rel intensity) 273 (100\%), 244 (34\%), HRMS (EI) m/e $\mathrm{C}_{17} \mathrm{H}_{11} \mathrm{~N}_{3} \mathrm{O}$ calcd. 273.0902, found 273.0903. (Literature: Fortunak, J. M. D.; Mastrocola, A. R.; Mellinger, M.; Sisti, N. J.; Wood, J. L.; Zhuang, Z.-P. Tetrahedron Lett. 1996, 37, 5679-5682).

2-Methoxy-7-methyl-9-oxo-9,11-dihydroindolizino[1,2-b]quinoline-8-carbonitrile 7b

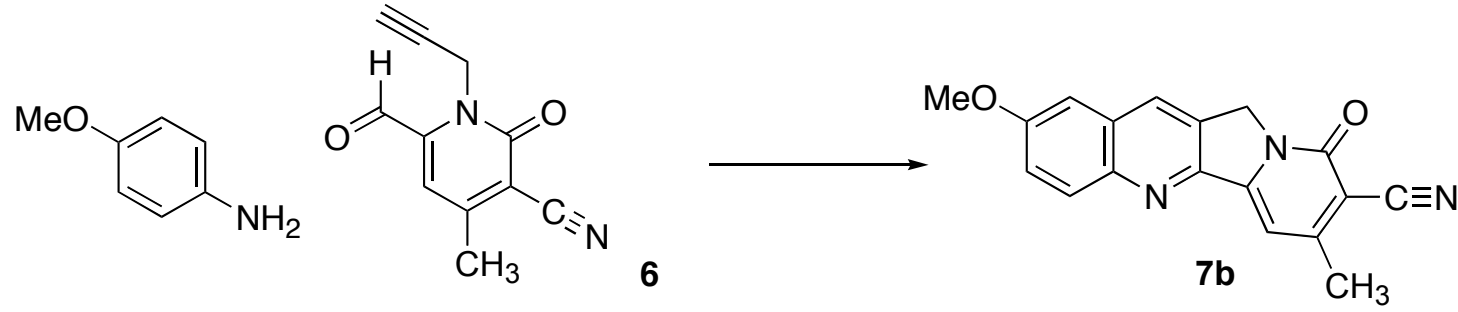

To 6-formyl-4-methyl-2-oxo-1-prop-2-ynyl-1,2-dihydro-pyridine-3-carbonitrile 6 (50 mg, 0.25 $\mathrm{mmol})$ in acetonitrile $(10 \mathrm{~mL})$ was added $p$-anisidine $(31 \mathrm{mg}, 0.25 \mathrm{mmol})$ followed by $\operatorname{Dy}(\mathrm{OTf})_{3}$ (15 mg, $0.025 \mathrm{mmol}$ ). The reaction mixture was heated at $50{ }^{\circ} \mathrm{C}$ for 16 hours, then allowed to 
cool. Removal of the solvent followed by recrystallization from hot methanol gave 2-methoxy-7methyl-9-oxo-9,11-dihydroindolizino[1,2-b]quinoline-8-carbonitrile $7 \mathbf{b}$ (49 $\mathrm{mg}, 64 \%)$ as a fine yellow powder. m.p. $\geq 300{ }^{\circ} \mathrm{C} ; \delta_{\mathrm{H}}\left(300 \mathrm{MHz}, \mathrm{CDCl}_{3}+\mathrm{CD}_{3} \mathrm{OD}\right) 8.39(1 \mathrm{H}, \mathrm{s}), 8.11(1 \mathrm{H}, \mathrm{d}, J=9.0$ $\mathrm{Hz}), 7.52(1 \mathrm{H}, \mathrm{m}), 7.33(1 \mathrm{H}, \mathrm{s}), 7.26(1 \mathrm{H}, \mathrm{m}), 5.31(2 \mathrm{H}, \mathrm{s}), 4.02(3 \mathrm{H}, \mathrm{s}), 2.67(3 \mathrm{H}, \mathrm{s}) ; \mathrm{MS}(\mathrm{EI})$ m/e (rel intensity) 303 (100\%), 260 (22\%); HRMS (EI) $m / e \mathrm{C}_{18} \mathrm{H}_{13} \mathrm{~N}_{3} \mathrm{O}_{2}$ calcd. 303.1008, found 303.1015. (Literature: Fortunak, J. M. D.; Mastrocola, A. R.; Mellinger, M.; Sisti, N. J.; Wood, J. L.; Zhuang, Z.-P. Tetrahedron Lett. 1996, 37, 5679-5682).

2-Amino- $N$-prop-2-ynylbenzamide 9<smiles>O=c1[nH]c2ccccc2c(=O)o1</smiles><smiles>C#CCNC(=O)c1ccccc1N</smiles>

To a stirred solution of isatoic anhydride $(7.90 \mathrm{~g}, 48 \mathrm{mmol})$ in DMF $(40 \mathrm{~mL})$ at $40-50{ }^{\circ} \mathrm{C}$, was added a solution of propargylamine $(4.98 \mathrm{~mL}, 73 \mathrm{mmol})$ in DMF $(20 \mathrm{~mL})$ dropwise. The resulting mixture was stirred at $40-50{ }^{\circ} \mathrm{C}$ for 3 hours, allowed to cool, and the DMF removed by rotary evaporation. Water $(200 \mathrm{~mL})$ was added and the $\mathrm{pH}$ adjusted to 9 using sodium hydroxide $(10 \mathrm{~mL}, 1.0 \mathrm{M})$. The resulting solid was extracted using $\mathrm{CH}_{2} \mathrm{Cl}_{2}(3 \times 100 \mathrm{~mL})$ and washed with brine $(1 \times 50 \mathrm{~mL})$. The combined organic extracts were dried over $\mathrm{MgSO}_{4}$, decolourized using charcoal, filtered and the solvent removed in vacuo to give 2-amino- $N$-prop-2-ynylbenzamide 9 $(5.47 \mathrm{~g}, 65 \%)$ as a white solid. m.p. $=98-100^{\circ} \mathrm{C}$; IR (film) 3426, 3289, 2252, 1638, 1618, 1587, $1529,1266,1159 \mathrm{~cm}^{-1} ; \delta_{\mathrm{H}}\left(300 \mathrm{MHz}, \mathrm{CDCl}_{3}\right) 7.33(1 \mathrm{H}, \mathrm{dd}, J=8.0,1.5 \mathrm{~Hz}), 7.22(1 \mathrm{H}$, ddd, $\mathrm{J}=$ 8.5, 7.5, 1.5 Hz), 6.69-6.61 (2H, m), 6.29 (1H, bs), $5.55(2 \mathrm{H}, \mathrm{bs}), 4.19(1 \mathrm{H}, \mathrm{dd}, J=5.0,2.5 \mathrm{~Hz})$, $2.27(1 \mathrm{H}, \mathrm{t}, J=2.5 \mathrm{~Hz}) ; \delta_{\mathrm{C}}\left(75 \mathrm{MHz}, \mathrm{CDCl}_{3}\right) 168.9,148.9,132.6,127.2,117.3,116.5,114.9$, 79.6, 71.7, 29.4; MS (EI) m/e (rel intensity) 174 (54\%), 120 (100\%), 119 (54\%), 92 (57\%); HRMS (EI) $m / e \mathrm{C}_{10} \mathrm{H}_{10} \mathrm{~N}_{2} \mathrm{O}$ calcd. 174.0793, found 174.0790. 
Acetic acid (2-prop-2-ynylcarbamoylphenylcarbamoyl)-methyl ester $\mathbf{1 0}$
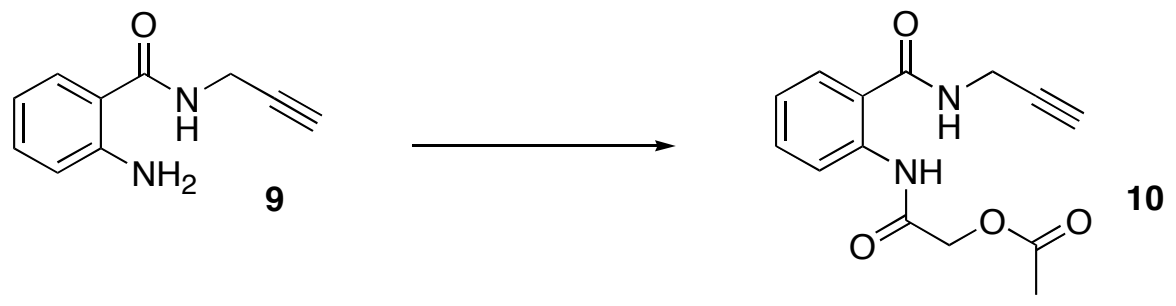

To a stirred solution of 2-amino- $N$-prop-2-ynylbenzamide $9(0.80 \mathrm{~g}, 4.60 \mathrm{mmol})$ and triethylamine $(962 \mu \mathrm{L}, 6.90 \mathrm{mmol})$ in benzene $(20 \mathrm{~mL})$, was added a solution of acetoxyacetyl chloride $(544 \mu \mathrm{L}, 5.06 \mathrm{mmol})$ in benzene $(4 \mathrm{~mL})$ dropwise at $40{ }^{\circ} \mathrm{C}$. After the reaction mixture was allowed to cool to room temperature and stand overnight, the solvent was removed under reduced pressure and the resulting residue was shaken with water $(10 \mathrm{~mL})$. The precipitate was collected by filtration and recrystallised (EtOH) to give acetic acid (2-prop-2ynylcarbamoylphenylcarbamoyl)-methyl ester $10(0.861 \mathrm{mg}, 68 \%)$ as a white solid. m.p. $=167$ $169{ }^{\circ} \mathrm{C}$; IR (film) 3273, 1741, 1686, 1626, 1596, 1526, 1448, 1227, 1055, $914 \mathrm{~cm}^{-1} ; \delta_{\mathrm{H}}(300$ $\left.\mathrm{MHz}, \mathrm{CDCl}_{3}\right) 11.62(1 \mathrm{H}, \mathrm{bs}), 8.61(1 \mathrm{H}, \mathrm{d}, J=8.0 \mathrm{~Hz}), 7.49(2 \mathrm{H}, \mathrm{m}), 7.11(1 \mathrm{H}, \mathrm{t}, J=7.5 \mathrm{~Hz})$, $6.57(1 \mathrm{H}, \mathrm{bs}), 4.71(2 \mathrm{H}, \mathrm{s}), 4.21(2 \mathrm{H}, \mathrm{dd}, J=5.0 \mathrm{~Hz}, 2.5 \mathrm{~Hz}), 2.33(3 \mathrm{H}, \mathrm{s}), 2.31(1 \mathrm{H}, \mathrm{t}, J=2.5$ $\mathrm{Hz}) ; \delta_{\mathrm{C}}\left(100 \mathrm{MHz}, \mathrm{CDCl}_{3}\right) 170.0,168.3,166.2,138.6,133.0,126.6,123.4,121.5,119.9,78.9$, 72.2, 62.8, 29.6, 20.7; MS (EI) m/e (rel intensity) 274 (19\%), 201 (100\%), 146 (75\%); HRMS (EI) $m / e \mathrm{C}_{14} \mathrm{H}_{14} \mathrm{~N}_{2} \mathrm{O}_{4}$ calcd. 274.0954, found 274.0953.

Acetic acid 4-prop-2-ynylimino-4H-benzo[d][1,3]oxazin-2-ylmethyl ester 11<smiles>C#CCNC(=O)c1ccccc1NC(=O)COC(C)=O</smiles><smiles>C#CCN=c1oc(COC(C)=O)nc2ccccc12</smiles>

To a stirred solution of acetic acid (2-prop-2-ynylcarbamoylphenylcarbamoyl)-methyl ester $\mathbf{1 0}$ (300 mg, $1.10 \mathrm{mmol})$ in $\mathrm{CH}_{2} \mathrm{Cl}_{2}(20 \mathrm{~mL})$, was added triphenylphosphine (1.44 g, $\left.5.47 \mathrm{mmol}\right)$, 
iodine $(1.39 \mathrm{~g}, 5.47 \mathrm{mmol})$ and Hünig's base $(1.89 \mathrm{~mL}, 10.9 \mathrm{mmol})$. The reaction mixture was stirred for 5 hours and quenched by the addition of sodium carbonate $(20 \mathrm{~mL}$, sat. aq. soln.) and extracted with $\mathrm{CH}_{2} \mathrm{Cl}_{2}(3 \times 30 \mathrm{~mL})$, dried $\left(\mathrm{MgSO}_{4}\right)$, filtered and concentrated under reduced pressure. Column chromatography (10\% EtOAc:Hexanes, pre-treated with $2 \%$ triethylamine) gave acetic acid 4-prop-2-ynylimino-4H-benzo[ $d][1,3]$ oxazin-2-ylmethyl ester 11 (250 mg, 89\%) as a light brown solid. m.p. $=97-99^{\circ} \mathrm{C} ; \mathrm{R}_{f}=0.66$ (50\% EtOAc:Hexanes); IR (film) 3243, 1735, $1681,1652,1462,1439,1259,1221,1046 \mathrm{~cm}^{-1} ; \delta_{\mathrm{H}}\left(300 \mathrm{MHz}, \mathrm{CDCl}_{3}\right) 8.12(1 \mathrm{H}, \mathrm{dd}, J=8.0,1.0$ $\mathrm{Hz}), 7.56(1 \mathrm{H}$, ddd, $J=9.0,7.0,1.5 \mathrm{~Hz}), 7.42-7.33(2 \mathrm{H}, \mathrm{m}), 4.94(2 \mathrm{H}, \mathrm{s}), 4.25(2 \mathrm{H}, \mathrm{d}, J=2.5$ $\mathrm{Hz}), 2.25(1 \mathrm{H}, \mathrm{t}, J=2.5 \mathrm{~Hz}), 2.22(3 \mathrm{H}, \mathrm{s}) ; \delta_{\mathrm{C}}\left(75 \mathrm{MHz}, \mathrm{CDCl}_{3}\right) 170.2,154.3,147.9,140.8$, 133.5, 128.7, 126.4, 126.1, 119.3, 81.2, 70.3, 61.4, 35.5 20.6; MS (EI) m/e (rel intensity) 256 (14\%), 183 (100\%), 84 (18\%); HRMS (EI) m/e $\mathrm{C}_{14} \mathrm{H}_{12} \mathrm{~N}_{2} \mathrm{O}_{3}$ calcd. 256.0848, found 256.0854.

Acetic acid 4-oxo-3-prop-2-ynyl-3,4-dihydroquinazolin-2-ylmethyl ester $\mathbf{1 2}$
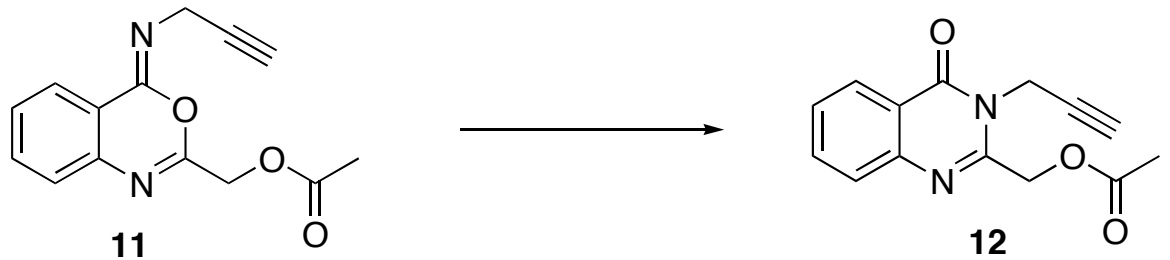

A solution of acetic acid 4-prop-2-ynylimino-4H-benzo[ $d][1,3]$ oxazin-2-ylmethyl ester 11 (300 $\mathrm{mg}, 1.20 \mathrm{mmol})$ in piperidine $(3.0 \mathrm{~mL})$ and ethylacetate $(12 \mathrm{~mL})$ was stirred for 1 hour at room temperature and then concentrated under reduced pressure. The crude imine was re-dissolved in ethyl acetate $(15 \mathrm{~mL}), \mathrm{SiO}_{2}(2.50 \mathrm{~g}, 42.0 \mathrm{mmol})$ was added and the reaction mixture stirred overnight. The suspension was filtered and the filtrate concentrated under reduced pressure. Flash chromatography (20\% EtOAc:Hexanes, pre-treated with 2\% triethylamine) gave acetic acid 4-oxo-3-prop-2-ynyl-3,4-dihydroquinazolin-2-ylmethyl ester 12 (256 $\mathrm{mg}, 85 \%)$ as a white solid. m.p. $=106-108^{\circ} \mathrm{C} ; \mathrm{R}_{f}=0.65$ (50\% EtOAc:Hexanes); IR (film) 3265, 1749, 1687, 1607, 1475, $1229,1041,774 \mathrm{~cm}^{-1} ; \delta_{\mathrm{H}}\left(400 \mathrm{MHz}, \mathrm{CDCl}_{3}\right) 8.29(1 \mathrm{H}, \mathrm{dd}, J=8.0,1.5 \mathrm{~Hz}), 7.77(1 \mathrm{H}, \mathrm{ddd}, J=$ 8.0, 7.0, $1.5 \mathrm{~Hz}), 7.70(1 \mathrm{H}, \mathrm{ddd}, J=8.0,1.5,0.5 \mathrm{~Hz}), 7.51(1 \mathrm{H}, \mathrm{ddd}, J=8.0,7.0,1.0 \mathrm{~Hz}), 5.33$ $(2 \mathrm{H}, \mathrm{s}), 4.96(2 \mathrm{H}, \mathrm{d}, J=2.5 \mathrm{~Hz}), 2.35(1 \mathrm{H}, \mathrm{t}, J=2.5 \mathrm{~Hz}), 2.23(3 \mathrm{H}, \mathrm{s}) ; \delta_{\mathrm{C}}\left(100 \mathrm{MHz}, \mathrm{CDCl}_{3}\right)$ 169.9, 161.0, 150.0, 146.6, 134.6, 127.7, 127.6, 127.0, 120.8, 77.2, 73.0, 63.8, 32.2, 20.7; MS 
(EI) m/e (rel intensity) 256 (35\%), 213 (77\%), 197 (100\%), 185 (28\%), 129 (30\%); HRMS (EI) m/e $\mathrm{C}_{14} \mathrm{H}_{12} \mathrm{~N}_{2} \mathrm{O}_{3}$ calcd. 256.0848, found 256.0851.

\section{2-Hydroxymethyl-3-prop-2-ynyl-3H-quinazolin-4-one}
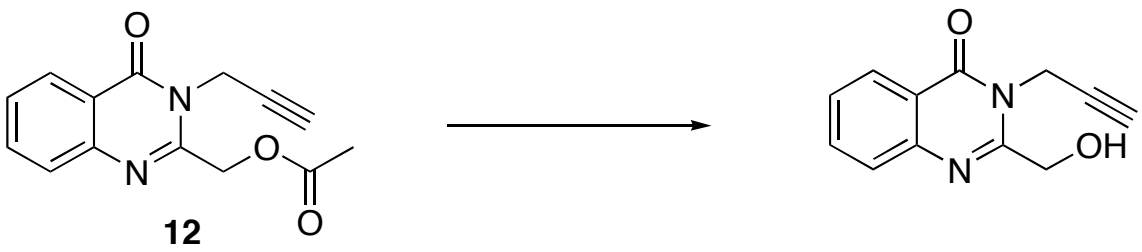

To a stirred solution of acetic acid 4-oxo-3-prop-2-ynyl-3,4-dihydroquinazolin-2-ylmethyl ester $12(600 \mathrm{mg}, 2.34 \mathrm{mmol})$ in THF (30 mL), was added sodium hydroxide solution (1.0 M, 4.69 $\mathrm{mL}, 4.69 \mathrm{mmol})$ dropwise, followed by water $(3.8 \mathrm{~mL})$. The reaction mixture was stirred for 1 hour and then concentrated under reduced pressure. The residue was treated with water $(40 \mathrm{~mL})$, the $\mathrm{pH}$ adjusted to 4.5 by the addition of $\mathrm{HCl}(1.0 \mathrm{M}, 4-5 \mathrm{~mL})$ and then extracted with ethyl acetate $(3 \times 40 \mathrm{~mL})$. The combined organic extracts were dried $\left(\mathrm{MgSO}_{4}\right)$ and concentrated under reduced pressure. Purification by column chromatography on silica (40\% EtOAc:Hexanes pretreated with $2 \%$ triethylamine) gave 2-hydroxymethyl-3-prop-2-ynyl-3 $H$-quinazolin-4-one (417 mg, 83\%) as a white solid. $\mathrm{R}_{f}=0.50$ (50\% EtOAc:Hexanes); m.p. $=126-128^{\circ} \mathrm{C}$; IR(film) $3425,1654,1609,1466,1427,1384,1266,1273,1024 \mathrm{~cm}^{-1} ; \delta_{\mathrm{H}}\left(400 \mathrm{MHz}, \mathrm{CDCl}_{3}\right) 8.25(1 \mathrm{H}, \mathrm{dd}$, $J=8.0,1.5 \mathrm{~Hz}), 7.76(1 \mathrm{H}, \mathrm{ddd}, J=8.0,7.0,1.5 \mathrm{~Hz}), 7.65(1 \mathrm{H}, \mathrm{d}, J=8.0 \mathrm{~Hz}), 7.48(1 \mathrm{H}, \mathrm{dd}, J=$ 8.0, $7.0 \mathrm{~Hz}), 4.84(2 \mathrm{H}, \mathrm{d}, J=2.5 \mathrm{~Hz}), 4.82(2 \mathrm{H}, \mathrm{s}), 2.64(1 \mathrm{H}, \mathrm{br} \mathrm{s}), 2.34(1 \mathrm{H}, \mathrm{t}, J=2.5 \mathrm{~Hz}) ; \delta_{\mathrm{C}}$ (100 MHz, $\left.\mathrm{CDCl}_{3}\right)$ 160.8, 154.3, 145.7, 134.7, 127.2, 127.1, 126.6, 120.3, 76.6, 73.1, 60.1, 30.7; MS (EI) m/e (rel intensity) 214 (52\%), 197 (39\%), 185 (39\%), 175 (100\%); HRMS (EI) m/e $\mathrm{C}_{12} \mathrm{H}_{10} \mathrm{~N}_{2} \mathrm{O}_{2}$ calcd. 214.0742, found 214.0741. 

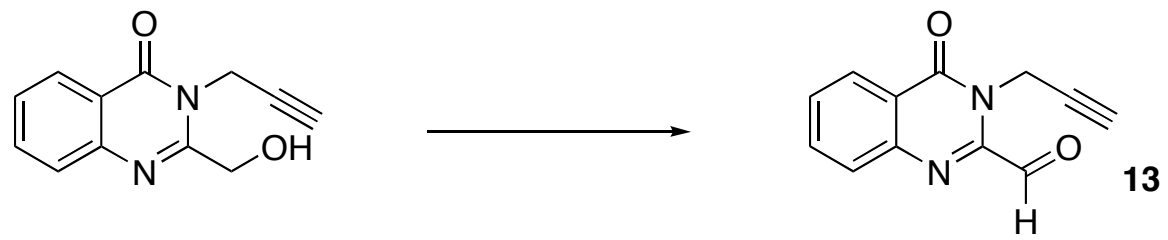

To a stirred solution of 2-hydroxymethyl-3-prop-2-ynyl-3H-quinazolin-4-one (100 mg, 0.467 mmol) and pyridine $(378 \mu \mathrm{L}, 4.67 \mathrm{mmol})$ in $\mathrm{CH}_{2} \mathrm{Cl}_{2}(30 \mathrm{~mL})$, was added Dess-Martin periodinane $(218 \mathrm{mg}, 0.514 \mathrm{mmol})$ at $0{ }^{\circ} \mathrm{C}$. The reaction mixture was stirred for 15 minutes at 0 ${ }^{\circ} \mathrm{C}$, followed by a further 1 hour at room temperature. Additional Dess-Martin periodinane (218 $\mathrm{mg}, 0.514 \mathrm{mmol}$ ) was added, and the reaction mixture stirred for 4 hours at room temperature. Filtration over Celite, removal of the solvent in vacuo, and column chromatography on silica (20\% EtOAc:Hexanes) gave 4-oxo-3-prop-2-ynyl-3,4-dihydroquinazolin-2-carbaldehyde 13 (77 mg, 78\%) as a white solid. m.p. $=169^{\circ} \mathrm{C}$ (decomp.); IR (film) 3423, 3053, 2986, 2305, 1724 , $1685,1420,1265 \mathrm{~cm}^{-1} ; \delta_{\mathrm{H}}\left(400 \mathrm{MHz}, \mathrm{CDCl}_{3}\right) 9.76(1 \mathrm{H}, \mathrm{s}), 8.37(1 \mathrm{H}, \mathrm{d}, J=7.5 \mathrm{~Hz}), 7.92-7.86$ $(2 \mathrm{H}, \mathrm{m}), 7.68(1 \mathrm{H}, \mathrm{ddd}, J=8.0,6.0,3.0 \mathrm{~Hz}) ; 5.40(2 \mathrm{H}, \mathrm{d}, J=2.5 \mathrm{~Hz}), 2.27(1 \mathrm{H}, \mathrm{t}, J=2.5 \mathrm{~Hz}) ; \delta_{\mathrm{C}}$ (75 MHz, $\left.\mathrm{CDCl}_{3}\right)$ 187.5, 160.5, 146.1, 145.9, 135.0, 130.2, 128.7, 127.4, 121.7, 78.1, 72.2, 31.5; MS (EI) m/e (rel intensity) 212 (49\%), 184 (100\%), 155 (27\%), 129 (36\%); HRMS (EI) m/e $\mathrm{C}_{12} \mathrm{H}_{8} \mathrm{~N}_{2} \mathrm{O}_{2}$ calcd. 212.0586, found 212.0591 .

\section{Luotonin A}
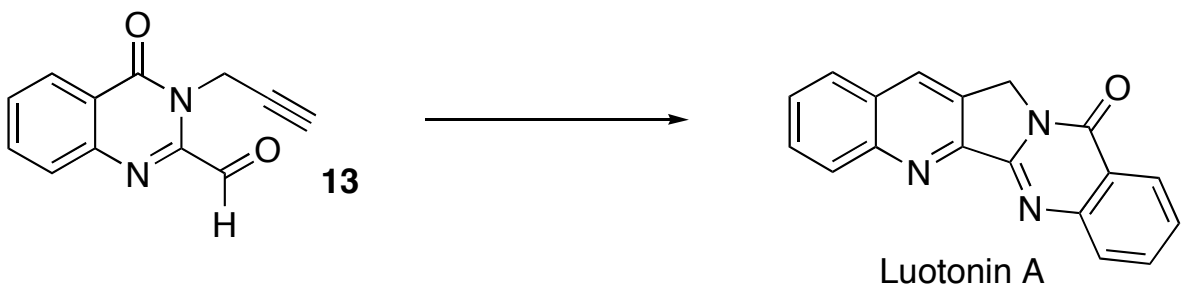

To 4-oxo-3-prop-2-ynyl-3,4-dihydroquinazolin-2-carbaldehyde 13 (50 $\mathrm{mg}, 0.236 \mathrm{mmol})$ in acetonitrile $(10 \mathrm{~mL})$ was added aniline $(22 \mu \mathrm{L}, 0.236 \mathrm{mmol})$ and Dy $(\mathrm{OTf})_{3}(15 \mathrm{mg}, 0.024 \mathrm{mmol})$. The reaction mixture was stirred at room temperature for 24 hours then water $(20 \mathrm{~mL})$ was added 
and the mixture extracted with $\mathrm{CH}_{2} \mathrm{Cl}_{2}(3 \times 30 \mathrm{~mL})$. The combined organic extracts were washed with brine $(1 \times 40 \mathrm{~mL})$, dried over $\mathrm{MgSO}_{4}$, filtered and the solvent removed in vacuo. Column chromatography on silica (20\% EtOAc:Hexanes) provided Luotonin A (67 mg, 51\%) as a pale yellow solid. m.p. $=278-280{ }^{\circ} \mathrm{C}$ (decomp.); IR (film) 3445, 3054, 2987, 2306, 1633, 1421, 1265 , $1018,896 \mathrm{~cm}^{-1} ; \delta_{\mathrm{H}}\left(500 \mathrm{MHz}, \mathrm{CDCl}_{3}+\mathrm{CD}_{3} \mathrm{OD}(3: 1)\right) 8.33(1 \mathrm{H}, \mathrm{s}) 8.17(2 \mathrm{H}, \mathrm{d}, J=8.5 \mathrm{~Hz}), 7.85$ $(1 \mathrm{H}, \mathrm{d}, J=8.0 \mathrm{~Hz}), 7.78(1 \mathrm{H}, \mathrm{d}, J=8.0 \mathrm{~Hz}), 7.67(2 \mathrm{H}, \mathrm{m}), 7.50(1 \mathrm{H}, \mathrm{t}, J=8.0 \mathrm{~Hz}), 7.40(1 \mathrm{H}, \mathrm{t}, J$ $=7.0 \mathrm{~Hz}), 5.16(2 \mathrm{H}, \mathrm{s}) ; \delta_{\mathrm{C}}\left(125 \mathrm{MHz}, \mathrm{CDCl}_{3}\right) 160.7,152.3,150.3,148.9$ (2 carbons), 134.5, 131.7, 130.5, 129.8, 129.3, 128.6, 128.3, 128.0, 127.8, 127.3, 125.9, 120.6, 47.1; MS (EI) m/e (rel intensity) 285 (100\%), 257 (15\%), 207 (19\%); HRMS (EI) m/e $\mathrm{C}_{18} \mathrm{H}_{11} \mathrm{~N}_{3} \mathrm{O}$ calcd. 285.0902, found 285.0900. (Literature: Mhaske, S. B.; Argade, N. P. J. Org. Chem. 2004, 69, 4563-4566 and references therein). 